\title{
Concomitant use of opioid medications with triptans or serotonergic antidepressants in US office-based physician visits
}

This article was published in the following Dove Press journal: Drug, Healthcare and Patient Safety

\section{Kyle C Molina \\ Kathleen A Fairman \\ David A Sclar}

Department of Pharmacy Practice, Midwestern University College of Pharmacy-Glendale, Glendale, AZ, USA
Correspondence: Kathleen A Fairman Department of Pharmacy Practice, Midwestern University College of Pharmacy-Glendale, 19555 N. 59th Avenue, Glendale, AZ 85308 USA Tel + I 6235723520

Email kfairm@midwestern.edu
Background: Opioids are not recommended for routine treatment of migraine because their benefits are outweighed by risks of medication overuse headache and abuse/dependence. A March 2016 US Food and Drug Administration (FDA) safety communication warned of the risk of serotonin syndrome from using opioids concomitantly with 5-hydroxytryptamine receptor agonists (triptans) or serotonergic antidepressants: selective serotonin reuptake inhibitors (SSRIs) or serotonin-norepinephrine reuptake inhibitors (SNRIs). Epidemiological information about co-prescribing of these medications is limited. The objective of this study was to estimate the nationwide prevalence of co-prescribing of an opioid with a serotonergic antidepressant and/ or triptan in US office-based physician visits made by 1) all patients and 2) patients diagnosed with migraine.

Methods: National Ambulatory Medical Care Survey (NAMCS) data were obtained for 2013 and 2014. Physician office visits that included the new or continued prescribing of $\geq 1$ opioid medication with a triptan or an SSRI/SNRI were identified. Co-prescribed opioids were stratified by agent to determine the proportion of co-prescriptions with opioids posing a higher risk of serotonergic agonism (meperidine, tapentadol, and tramadol).

Results: Of an annualized mean 903.6 million office-based physician visits in 2013-2014, 17.7 million ( $2.0 \%$ of all US visits) resulted in the prescribing of $\geq 1$ opioid medication with a triptan or an SSRI/SNRI. Opioid-SSRI/SNRI was co-prescribed in 16,044,721 visits, while opioid-triptan was co-prescribed in 1,622,827 visits. One-fifth of opioid co-prescribing was attributable to higher-risk opioids, predominantly tramadol (18.6\% of opioid-SSRI/SNRI, $21.8 \%$ of opioid-triptan). Of 7,672,193 visits for patients diagnosed with migraine, $16.3 \%$ included opioid prescribing and $2.0 \%$ included co-prescribed opioid-triptan.

Conclusion: During a period approximately 2 years prior to an FDA warning about the risk of serotonin syndrome from opioid-SSRI/SNRI or opioid-triptan co-prescribing, use of these combinations was common in the USA. Studies on prescribing patterns following the March 2016 warning, and on the risk of serotonin syndrome associated with these co-prescriptions, are needed.

Keywords: serotonin syndrome, medication safety, FDA adverse event reporting system, SNRI, SSRI

\section{Introduction}

American Academy of Family Physicians (AAFP) and American Headache Society (AHS) guidelines for the treatment of acute migraine headache recommend against routine use of opiate-containing compounds for the abortive treatment of migraine. ${ }^{1,2}$ This recommendation is based on concerns about dependence and medication 
overuse headache. ${ }^{1,2}$ Guideline-recommended treatments include non-steroidal anti-inflammatory drugs (NSAIDs), acetaminophen combinations, 5-hydroxytryptamine receptor agonists (triptans), ergot alkaloids, and antiemetics. ${ }^{1,2}$ Similar concerns about the misuse or overuse of opioids for other conditions led to the development of US Centers for Disease Control and Prevention guidelines for treatment of chronic, non-cancer pain, which recommend the use of nonopioid modalities. ${ }^{3,4}$

In addition to the concerns raised in these guidelines, use of opioids for the treatment of migraine can lead to serious adverse drug events. In March 2016, the US Food and Drug Administration (FDA) issued a safety communication warning that opioid pain medications may interact with antidepressant and migraine medications, resulting in a serotonin syndrome, a rare but potentially life-threatening serious central nervous system reaction caused by excess serotonergic agonism. ${ }^{5}$ This safety communication came almost 10 years after a similar warning in July 2006, regarding the risk of serotonin syndrome with concomitant use of triptans and the serotonergic antidepressants: selective serotonin reuptake inhibitors (SSRIs) or serotonin-norepinephrine reuptake inhibitors (SNRIs). ${ }^{6}$ Both FDA warnings were based on analyses of adverse events reported to the FDA. ${ }^{5,6}$

Opioids may differ in their propensity to cause excess serotonergic agonism, although lists of higher-risk opioids vary somewhat, depending on their source. Prior to the March 2016 warning, 3 opioid products - meperidine, tapentadol, and tramadol - carried labeled warnings for risk of serotonin syndrome. ${ }^{5}$ Three phenylpiperidine opioids - meperidine, methadone, and tramadol - are known inhibitors of serotonin reuptake, and a 2016 literature review indicated that these are the most commonly reported opioids in cases of serotonin syndrome. ${ }^{7,8}$ The March 2016 FDA adverse events analysis, which did not include meperidine, tapentadol, or tramadol, concluded that the opioids most commonly associated with serotonin syndrome were fentanyl, methadone, and oxycodone. ${ }^{5}$

Despite AAFP and AHS guideline recommendations, opioids remain a common treatment modality for patients with migraine, who may be disproportionate users of medication combinations associated with elevated risk of serotonin syndrome. An evaluation of 3 emergency departments, published in 2017 , found that opioids were ordered in $35.8 \%$ of visits for migraines, either as first-line or rescue treatment. ${ }^{9}$ An analysis of data from the 2009 American Migraine Prevalence and Prevention (AMPP) study found that $15.9 \%$ of persons with migraine were current users of opioids, and $13.8 \%$ were previous users of opioids. ${ }^{10}$

Elevated risk of serotonin syndrome may also result from comorbidities. In the AMPP study, patients with migraine who were using opioids had a higher prevalence of depression and anxiety than did those not using opioids. ${ }^{10}$ As serotonergic antidepressants and opioids rank as the 2 most commonly reported medication classes that contribute to serotonin syndrome, this pattern of medication use puts patients treated with opioids for migraine at higher risk of serotonin syndrome, as they may be prescribed SSRI or SNRI to treat their depressive symptoms. ${ }^{11}$

In an analysis of National Ambulatory Medical Care Survey (NAMCS) data for 2007-2008, Sclar et al found that of patients prescribed either a triptan or a serotonergic antidepressant, $1.8 \%$ were prescribed both agents. ${ }^{12}$ Since that time, no publications of which these authors are aware have examined the prevalence of use of medication combinations that carry elevated risk of serotonin syndrome. The present study was designed to provide updated estimates of the nationwide prevalence of combined use of an opioid with a serotonergic antidepressant and/or triptan in communitybased care provided in US physician offices.

\section{Methods \\ Data source}

Data were obtained from the NAMCS, a cross-sectional, nationally representative survey of the care provided in office-based physician visits that is conducted annually by the National Center for Health Statistics (NCHS). ${ }^{13,14}$ Available data for each office visit include patient demographics, payment source, diagnoses (up to 3 diagnoses until 2013 and up to 5 diagnoses beginning in 2014), chronic comorbidities, procedures and treatments, and a list of all medications newly prescribed or continued. All data are abstracted from patient medical records, primarily by employees of the US Bureau of the Census and, less commonly, by physicians or office staff. ${ }^{13}$ All NAMCS data sets are publicly available for download from the NCHS website and contain no identifiable information about either patients or physicians. Thus, no institutional review board exemption or approval was sought, as is typical of published studies that use NAMCS data. ${ }^{15-17}$

The NAMCS employs a multistage probability sampling procedure comprising the following steps: 1 ) initial selection of geographic areas (eg, counties, towns); 2) selection of a random sample of physicians, stratified by specialty, using lists obtained from the American Medical Association and 
the American Osteopathic Association; 3) random assignment of each physician to 1 of 52 weeks in the calendar year; and 4) systematic random sampling of all visits during the assigned week. ${ }^{14}$ In 2014, the most recent year for which NAMCS data were available at the time of the present study, the survey response rate was $55 \% .{ }^{13}$ For each office visit, the NAMCS record includes a weight that adjusts for the complex sampling design and for survey non-response. ${ }^{14}$ Application of the weight to the data yields nationally representative estimates of care provided in all US office-based physician visits during the calendar year. ${ }^{18}$

\section{Sample}

The study sample included all office visits made in 2013 or 2014. In accordance with guidance provided by the NCHS, and with previous uses of the data set, two years of data were analyzed to enhance the sample size and reliability of the estimates. ${ }^{12,19}$

For each office visit provided in either 2013 or 2014, the first 10 listed medications that were either prescribed or continued were chosen for analysis. From that list, triptans, serotonergic antidepressants, and opioids were identified (Table S1). All formulations and routes (eg, oral tablets, extended-release, nasal spray, and patches) were included. Visits were then grouped by combination type: opioids with triptans, opioids with serotonergic antidepressants, and all 3 classes.

\section{Descriptive metrics}

Counts and percentages of office visits that included each combination of the 3 therapy class groups were calculated. To summarize across the 2 years of data, counts were calculated as annualized means (ie, weighted counts, summed and divided by 2 ). In addition, for each identified therapy class combination, profiles were constructed based on patient demographics, payer type, and provider specialty. Diagnosis of migraine was measured as an ICD-9 code of 346.xx in any of the first 3 listed diagnosis fields.

Because the risk of serotonin syndrome associated with opioids may differ by medication, the rates of opioid coprescription with triptans and serotonergic antidepressants were calculated for individual medications. Higher-risk medications were identified as those that carried an FDA warning for serotonin syndrome prior to 2016: meperidine, tapentadol, and tramadol. ${ }^{5}$

All reported results represent national population estimates derived from the application of the weights provided by NCHS to the individual visit data. No statistical tests were performed. All analyses were performed using SPSS version 23.0 (IBM Corp., Armonk, NY, USA).

\section{Results}

Of an annualized average of 903.6 million office-based physician visits in the USA in 2013-2014, 192,137,974 (21.2\%) included the prescribing of $\geq 1$ of the 3 study medication classes: opioids, serotonergic antidepressants, or triptans. A migraine diagnosis was made in 7,672,193 visits $(0.8 \%)$. Of visits made by patients diagnosed with migraine, a triptan was prescribed in $28.7 \%$, an opioid in $16.3 \%$, both opioids and SSRI/SNRI in $3.8 \%$, and both opioids and triptans in $2.0 \%$ (Table 1).

Opioids were co-prescribed with serotonergic antidepressants at more than 16 million visits annually, representing $1.8 \%$ of all office visits made in 2013-2014 and $15.5 \%$ of visits at which $\geq 1$ opioid was prescribed $(n=103,282,200)$ (Table 1). Co-prescribing of opioids with triptans was much less common, occurring in only $0.2 \%$ of visits overall and $1.6 \%$ of visits that included the prescribing of $\geq 1$ opioid. However, co-prescribed opioid-triptan represented 21.2\% of visits at which a triptan was prescribed. Co-prescribing of serotonergic antidepressants, opioids, and triptans occurred at $0.05 \%$ of total office visits (profiles not shown in Table 1 because unweighted $n=37$ visits). In total, opioid co-prescription with a triptan or an SSRI/SNRI occurred at $17,667,548$ visits (2.0\% of all US visits).

Of visits that included co-prescribing of opioids with either a serotonergic antidepressant or a triptan, $20.0 \%$ involved the prescribing of an opioid that had a warning label for serotonin syndrome prior to March 2016 (Table 2). Predominant among these was tramadol, which was prescribed in $21.8 \%$ of opioid-triptan visits and $18.6 \%$ of opioid-SSRI/ SNRI visits. Prescribing of meperidine (2.4\% of opioid-triptan visits; $0.8 \%$ of opioid-SSRI/SNRI antidepressant visits) and tapentadol $(0.5 \%$ of opioid-triptan visits; $0.3 \%$ of opioid-SSRI/SNRI visits) was much less common.

\section{Discussion}

This analysis of office-based physician visits provided to patients in 2013-2014 produced several potentially concerning findings about the prescribing of opioid medications, overall and to patients with migraine. Although comprising only a small fraction of total US office visits $(2.0 \%)$, an estimated 17,667,548 office visits annually resulted in opioid co-prescribing that posed potentially increased risk of serotonin syndrome (ie, either opioid-triptan or opioid-SSRI/ SNRI). These co-prescriptions represented $17.1 \%$ of all visits 
Table I Characteristics of patients prescribed serotonergic antidepressants, opioids, and/or triptans, ${ }^{a}$ US office visits in $2013-2014$

\begin{tabular}{|c|c|c|}
\hline & SSRI/SNRI and opioid & Triptan and opioid \\
\hline \multicolumn{3}{|c|}{ Office visit counts, total and by medication prescribed or continued } \\
\hline $\mathrm{N}$ unweighted office visits & 1,923 & 217 \\
\hline $\mathrm{N}$ weighted office visits (annualized) ${ }^{\mathrm{b}}$ & $|6,044,72|$ & $1,622,827$ \\
\hline $\mathrm{N}$ weighted office visits (annualized) ${ }^{\mathrm{b}}$ with migraine diagnosis ${ }^{c}$ & 294,635 & 153,370 \\
\hline Percentage of total US office visits & 1.8 & 0.2 \\
\hline Percentage of visits at which $\geq I$ opioid was prescribed & 15.5 & 1.6 \\
\hline Percentage of visits at which $\geq I$ triptan was prescribed & N/A & 21.2 \\
\hline Patient characteristics, $n(\%)$ of visits & $|6,044,72|(100.0)$ & $1,622,827(100.0)$ \\
\hline \multicolumn{3}{|l|}{ Gender } \\
\hline Male & $4,671,094(29.1)$ & $318,324(19.6)$ \\
\hline Female & $11,373,627(70.9)$ & $\mathrm{I}, 304,503(80.4)$ \\
\hline \multicolumn{3}{|l|}{ Age (years) } \\
\hline$<20$ & I I $2,872(0.7)$ & $0(0.0)$ \\
\hline $20-39$ & $2,082,948(13.0)$ & $338,615(20.9)$ \\
\hline $40-59$ & $7,015,937(43.7)$ & $960,37 \mid(59.2)$ \\
\hline$\geq 60$ & $6,832,964(42.6)$ & $323,842(20.0)$ \\
\hline \multicolumn{3}{|l|}{ Ethnicity, n (\%) } \\
\hline White, non-Hispanic & $13,580,306(84.6)$ & I,32I,4II (8I.4) \\
\hline Black, non-Hispanic & $883,55 I(5.5)$ & $|35,78|(8.4)$ \\
\hline Hispanic & $1,227,438(7.7)$ & $|5|, 810(9.4)$ \\
\hline Others & $353,426(2.2)$ & $13,825(0.9)$ \\
\hline \multicolumn{3}{|l|}{ Physician specialty } \\
\hline General/family practice & $5,576,119(34.8)$ & $652,115(40.2)$ \\
\hline Internal medicine & $3,171,979(19.8)$ & $177,033(10.9)$ \\
\hline Neurology & $316,642(2.0)$ & $66,840(4.1)$ \\
\hline Psychiatry & $369,895(2.3)$ & $5,755(0.4)$ \\
\hline Others & $6,610,087(41.1)$ & $721,085(44.4)$ \\
\hline
\end{tabular}

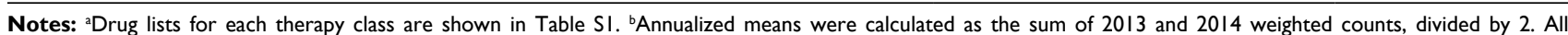
percentages shown in the table were based on the annualized mean counts unless otherwise specified. ${ }^{\mathrm{C}}$ Total annualized weighted $\mathrm{N}$ of all office visits made by patients with migraine=7,672,193; of these, 3.8\% ( $n=294,635)$ included co-prescribed SSRI/SNRI with opioids, and 2.0\% ( $n=153,370)$ included co-prescribed triptan with opioids.

Abbreviations: N/A, not applicable; SNRI, serotonin-norephinephrine reuptake inhibitor; SSRI, selective serotonin reuptake inhibitor; triptan, 5-hydroxytryptamine receptor agonist.

Table 2 Specific opioid agents prescribed with triptan or SSRI/SNRI, US office visits in 2013-2014

\begin{tabular}{|c|c|c|}
\hline & \multicolumn{2}{|c|}{ Medications prescribed or continued } \\
\hline & Opioid and SSRI/SNRI & Opioid and triptan \\
\hline $\mathbf{N}$ weighted office visits (annualized) ${ }^{a}$ & $|6,044,72|$ & $1,622,827$ \\
\hline \multicolumn{3}{|l|}{ Opioid medication, $\mathrm{n}(\%)$ of visits ${ }^{\mathrm{b}}$} \\
\hline Buprenorphine & $727,686(4.5)$ & $65,938(4.1)$ \\
\hline Butorphanol & $4,542(0.0)$ & $0(0.0)$ \\
\hline Codeine & $1,086,838(6.8)$ & $123,083(7.6)$ \\
\hline Dihydrocodeine & $0(0.0)$ & $6,871(0.4)$ \\
\hline Fentanyl & $742,605(4.6)$ & $36,597(2.3)$ \\
\hline Hydrocodone & $7,478,472(46.6)$ & $719,063(44.3)$ \\
\hline Hydromorphone & $418,185(2.6)$ & $27,275(1.7)$ \\
\hline Meperidine $^{c}$ & $127,218(0.8)$ & $38,646(2.4)$ \\
\hline Methadone & $431,105(2.7)$ & $7,885(0.5)$ \\
\hline Morphine & $766,833(4.8)$ & $64,310(4.0)$ \\
\hline Oxycodone & $3,786,678(23.6)$ & $429,680(26.5)$ \\
\hline Oxymorphone & $35,032(0.2)$ & $0(0.0)$ \\
\hline Tapentadolc & $46,762(0.3)$ & $7,723(0.5)$ \\
\hline Tramadolc $^{c}$ & $2,985,378(18.6)$ & $353,950(21.8)$ \\
\hline Any medication with FDA warning prior to March 2016 & $3,159,358(19.7)$ & $400,320(24.7)$ \\
\hline
\end{tabular}

Notes: ${ }^{a}$ Annualized means were calculated as the sum of 2013 and 2014 weighted counts, divided by $2 .{ }^{\text {b } A l f e n t a n i l, ~ p e n t a z o c i n e, ~ a n d ~ s u f e n t a n i l ~ a r e ~ n o t ~ s h o w n ~ b e c a u s e ~ t h e y ~}$ were prescribed on no (zero) visits. 'An opioid whose label contained a warning for risk of serotonin syndrome prior to March 2016. All percentages shown in the table were based on the annualized mean counts.

Abbreviations: FDA, US Food and Drug Administration; SNRI, serotonin-norephinephrine reuptake inhibitor; SSRI, selective serotonin reuptake inhibitor; triptan, 5-hydroxytryptamine receptor agonist. 
in which $\geq 1$ opioid was prescribed. Among patients with a diagnosis of migraine, $16.3 \%$ received an opioid, reflecting little change in the use of opioids in patients with migraine since the 2009 AMPP study. ${ }^{10}$

Of note, our 2013-2014 study period precedes by approximately 2 years the March 2016 FDA warning about the risk of serotonin syndrome with opioids. In addition, the majority of the present study period preceded the Drug Enforcement Agency (DEA) reclassifications of tramadol as a schedule IV controlled substance and of hydrocodone as a schedule II controlled substance, which took effect in August 2014 and October 2014, respectively. ${ }^{20,21}$ Additional study of a later period is needed to determine how these FDA and DEA actions affect the prescribing of these agents.

In this context, it should be noted that as providers become aware of the 2016 warning about excessive serotonin agonism associated with opioid-SSRI/SNRI and opioid-triptan combinations, prescribing may change. However, the finding that tramadol predominated among the opioid combinations observed in the present study, despite its warning label at that time, suggests that prescribers may lack awareness of the risk of excess serotonin agonism or perceive this risk to be negligible in their clinical decision making. ${ }^{22}$ This finding is of particular interest, as tramadol has been reportedly linked to a greater number of cases of serotonin syndrome than have other opioid agents. ${ }^{8}$ As most cases of serotonin syndrome produce only mild symptomatology, some observers have suggested a continuum of excess serotonergic agonism, reserving the diagnostic label of "serotonin syndrome" for severe cases. ${ }^{8}$

There are several important limitations to our study, many of which are intrinsic to use of the NAMCS. First, NAMCS drug fields indicate only medication, not quantity or strength. Higher-dosage treatment with opioids, particularly by patients misusing prescribed medications, may increase the risk of excess serotonin agonism but could not be measured in the present study.

Second, the NAMCS reports medications that were prescribed or continued by a physician at an office visit, and not whether the medication is filled or taken by the patient. Therefore, many patients in this study prescribed a combination with elevated risk of serotonin syndrome may not actually be at risk of this drug-drug interaction. It is, however, important to note that the use of a single serotonergic agent still may pose a risk to patients. An analysis of a large French pharmacovigilance database demonstrated that nearly onehalf of the reported cases of serotonin syndrome resulted from the use of a single serotonergic agent. ${ }^{11}$ Consequently, a patient taking any one of these medications may be at elevated risk of developing serotonin syndrome. However, the difference in absolute risk in developing serotonin syndrome, comparing use of 1 versus 2 serotonergic agents, is not known at this time.

A related third limitation is that this study did not specifically assess the rate of serotonin syndrome; rather, it is a study of potential risk based on medication product labels. Despite case reports of serotonin syndrome, the actual risk of serotonin syndrome with use of serotonergic antidepressants, opioids, and triptans remains unknown because of a lack of population-based prevalence analyses. The updated labeling by the FDA came after a review of adverse events found only 43 reported cases of serotonin syndrome from January 1, 1969, to June 12, 2013. In that regard, the 2016 FDA action was similar to its 2006 alert regarding the use of triptan agents with serotonergic antidepressants, which was based on only 29 reported cases of serotonin syndrome. A review of these 29 cases found that only 7 met the Sternbach criteria for diagnosis of serotonin syndrome, while no cases met the Hunter criteria. ${ }^{23}$ A 2016 review corroborates the infrequency of serotonin syndrome, as events related to opioid use were reported in fewer than 40 manuscripts published between 1974 and December 2015. ${ }^{8}$ Thus, the incidence of serotonin syndrome may be exceedingly small. However, serotonin syndrome is likely to be underreported owing to challenging differential diagnosis and highly variable severity, and the FDA adverse event database captures only reported cases.

Our findings may appropriately be interpreted in light of the broader public health concern about prescribing of opioids, the rate of which approximately tripled from 1999 to 2015 in the USA. ${ }^{24}$ Findings of the present study theoretically suggest growth in risks of adverse effects, potentially including serotonin syndrome, associated with this increase in opioid use. Countering the policy implications of this potentially increased risk, it has been suggested that warnings about prescribed medications may lead to unintended consequences. For example, one analysis found that the FDA warning in 2004 regarding the risk of antidepressantrelated suicide in adolescents and young adults contributed to undertreatment of depression, resulting in an increased rate of suicidal behavior. ${ }^{25}$ Therefore, treatment with serotonergic antidepressants in patients with migraine and comorbid depression must not be unnecessarily discouraged, given the importance of treatment with appropriate pharmacotherapy and evidence that depression is highly prevalent and may be undertreated in this patient population. ${ }^{26}$ 
Nonetheless, in prescribing pharmacotherapy for migraine and other chronic pain disorders, health care providers should consider the risks associated with opioid use, particularly when co-prescribed with other serotonergic medications. The prescribing of opioids comes with the need to balance the risks of abuse and addiction against the need for pain control. ${ }^{27}$ This need is especially acute in the treatment of migraine, a highly debilitating and painful disorder for which patients need pharmacotherapy that is both effective and safe.

\section{Acknowledgment}

The study was funded by Midwestern University, with no external sources of funding or support.

\section{Disclosure}

$\mathrm{KCM}, \mathrm{KAF}$, and DAS report no financial or other conflicts of interest related to this work.

\section{References}

1. Gilmore B, Michael M. Treatment of acute migraine headache. Am Fam Physician. 2011;83(3):271-280.

2. Marmura MJ, Silberstein SD, Schwedt TJ. The acute treatment of migraine in adults: the american headache society evidence assessment of migraine pharmacotherapies. Headache. 2015;55(1):3-20.

3. Dowell D, Haegerich TM, Chou R. CDC Guideline for prescribing opioids for chronic pain - United States, 2016. MMWR Recomm Rep. 2016;65(1):1-49.

4. Schuchat A, Houry D, Guy GP Jr. New data on opioid use and prescribing in the United States. JAMA. 2017;318(5):425-426.

5. US Food and Drug Administration [homepage on the Internet]. FDA Drug Safety Communication: FDA warns about several safety issues with opioid pain medicines; requires label changes. 2016 [March 22]. Available from: https://www.fda.gov/Drugs/DrugSafety/ucm489676. htm. Accessed August 19, 2017.

6. US Food and Drug Administration [homepage on the Internet]. Information for healthcare professionals: selective serotonin reuptake inhibitors (SSRIs), selective serotonin-norepinephrine reuptake inhibitors (SNRIs), 5-hydroxytryptamine receptor agonists (triptans). 2006 July [updated 2013, August 14]. Available from: https://wayback. archive-it.org/7993/20170112170458/http://www.fda.gov/Safety/ MedWatch/SafetyInformation/SafetyAlertsforHumanMedicalProducts/ ucm150367.htm. Accessed August 19, 2017.

7. Gillman PK. Monoamine oxidase inhibitors, opioid analgesics and serotonin toxicity. Br J Anaesth. 2005;95(4):434-441.

8. Ansari H, Kouti L. Drug interaction and serotonin toxicity with opioid use: another reason to avoid opioids in headache and migraine treatment. Curr Pain Headache Rep. 2016;20(8):50.

9. Young N, Silverman D, Bradford H, Finkelstein J. Multicenter prevalence of opioid medication use as abortive therapy in the emergency department treatment of migraine headaches. Am J Emerg Med. 2017;35(12):1845-1849.

10. Buse DC, Pearlman SH, Reed ML, Serrano D, Ng-Mak DS, Lipton RB. Opioid use and dependence among persons with migraine: results of the AMPP study. Headache. 2012;52(1):18-36.
11. Abadie D, Rousseau V, Logerot S, Cottin J, Montastruc JL, Montastruc F. Serotonin syndrome: analysis of cases registered in the French pharmacovigilance database. J Clin Psychopharmacol. 2015;35(4): 382-388.

12. Sclar DA, Robison LM, Castillo LV, et al. Concomitant use of triptan, and SSRI or SNRI after the US Food and Drug Administration Alert on serotonin syndrome. Headache. 2012;52(2):198-203.

13. US Centers for Disease Control and Prevention NCHS. 2014 NAMCS micro-data file documentation. 2014. Available from: https://ftp.cdc.gov/ pub/health_statistics/nchs/dataset_documentation/NAMCS/doc2014. pdf. Accessed August 15, 2017.

14. US Centers for Disease Control and Prevention, National Center for Health Statistics [homepage on the Internet]. NAMCS scope and sample design. 2010 [January 15]. Available from: https://www.cdc.gov/nchs/ ahcd/ahcd_scope.htm. Accessed August 19, 2017.

15. US Centers for Disease Control and Prevention, National Center for Health Statistics [homepage on the Internet]. NAMCS, 1993-2015. 2017 [December 18]. Available from: ftp://ftp.cdc.gov/pub/Health_Statistics/ NCHS/Datasets/NAMCS. Accessed January 12, 2018.

16. Prunuske JP, St Hill CA, Hager KD, et al. Opioid prescribing patterns for non-malignant chronic pain for rural versus non-rural US adults: a population-based study using 2010 NAMCS data. BMC Health Serv Res. 2014;14:563.

17. Mafi JN, Edwards ST, Pedersen NP, Davis RB, McCarthy EP, Landon BE. Trends in the ambulatory management of headache: analysis of NAMCS and NHAMCS data 1999-2010. J Gen Intern Med. 2015;30(5): 548-555.

18. US Centers for Disease Control and Prevention, National Center for Health Statistics [homepage on the Internet]. NAMCS estimation procedures. 2010 [January 15]. Available from: https://www.cdc.gov/nchs/ ahcd/ahcd_estimation_procedures.htm. Accessed August 31, 2017.

19. Cherry D. Understanding and using NAMCS and NHAMCS data: data tools and basic programming techniques. 2008. Available from: http:// slideplayer.com/slide/8713172/. Accessed August 14, 2017.

20. US Government Publishing Office [homepage on the Internet]. Schedules of controlled substances: placement of tramadol into Schedule IV. Federal Register; 2014 [July 2]. Available from: https://www.gpo.gov/ fdsys/pkg/FR-2014-07-02/pdf/2014-15548.pdf. Accessed August 31, 2017.

21. US Department of Justice, Drug Enforcement Administration [homepage on the Internet]. Final rule: rescheduling of hydrocodone combination products from Schedule III to Schedule II. Federal Register; 2014 [August 22]. Available from: https://www.deadiversion.usdoj.gov/ fed_regs/rules/2014/fr0822.htm. Accessed August 31, 2017.

22. Tepper SJ. Serotonin syndrome: SSRIs, SNRIs, triptans, and current clinical practice. Headache. 2012;52(2):195-197.

23. Evans RW. The FDA alert on serotonin syndrome with combined use of SSRIs or SNRIs and triptans: an analysis of the 29 case reports. MedGenMed. 2007;9(3):48.

24. US Centers for Disease Control and Prevention [homepage on the Internet]. Opioid prescribing is still high and varies widely throughout the U.S. CDC Newsroom Releases; 2017 [July 6]. Available from: https://www.cdc.gov/media/releases/2017/p0706-opioid.html. Accessed August 31, 2017.

25. Lu CY, Zhang F, Lakoma MD, et al. Changes in antidepressant use by young people and suicidal behavior after FDA warnings and media coverage: quasi-experimental study. BMJ. 2014;348:g3596.

26. Amoozegar F, Patten SB, Becker WJ, et al. The prevalence of depression and the accuracy of depression screening tools in migraine patients. Gen Hosp Psychiatry. 2017;48:25-31.

27. Kotalik J. Controlling pain and reducing misuse of opioids: ethical considerations. Can Fam Physician. 2012;58(4):381-385. 


\section{Supplementary material}

Table SI Identified medications by therapy class

Opioids

Alfentanil, buprenorphine, butorphanol, codeine, and all codeine combination products, ${ }^{\text {a }}$ dihydrocodeine combinations; fentanyl, hydrocodone, and hydrocodone combinations including hydrocodone/acetaminophen and cough/cold products, hydromorphone, meperidine, meperidine/promethazine combinations; methadone, morphine, oxycodone, oxymorphone, naloxone/pentazocine, remifentanil, sufentanil, tapentadol, tramadol, tramadol/acetaminophen

Serotonergic Citalopram, desvenlafaxine, duloxetine, escitalopram, fluoxetine, fluoxetine/olanzapine combination, fluvoxamine, antidepressants levomilnacipran, paroxetine, sertraline, venlafaxine

Triptans Almotriptan, eletriptan, frovatriptan, naratriptan, rizatriptan, sumatriptan, sumatriptan/naproxen combination, zolmitriptan

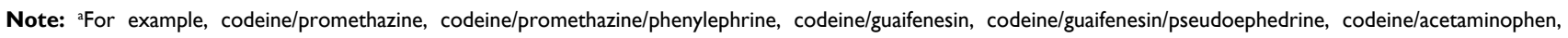
codeine/aspirin.

Abbreviation: triptans, 5-hydroxytryptamine receptor agonists.

\section{Publish your work in this journal}

Drug, Healthcare and Patient Safety is an international, peer-reviewed open access journal exploring patient safety issues in the healthcare continuum from diagnostic and screening interventions through to treatment, drug therapy and surgery. The journal is characterized by the rapid reporting of reviews, original research, clinical, epidemiological and post-marketing surveillance studies, risk management, health literacy and educational programs across all areas of healthcare delivery. The manuscript management system is completely online and includes a very quick and fair peer-review system. Visit http://www.dovepress.com/ testimonials.php to read real quotes from published authors.

Submit your manuscript here: https://www.dovepress.com/drug-healthcare-and-patient-safety-journal 